\section{ARP3 in podocyte development}

Podocytes are highly differentiated cells that have a complex cytoskeletal architecture. They extend numerous protrusions - primary processes and terminally branched protrusions called secondary (or foot) processes - which interdigitate and anchor the podocyte to the glomerular basement membrane. Appropriate formation of podocyte processes is critical for the functioning of the glomerular filtration barrier; however, the pathways underlying podocyte process formation are unclear. New research has identified a key role for the N-WASP-ARP2-ARP3 axis in regulating podocyte morphology. "Analyses revealed that ARP3 has an important role in the development of secondary processes," say Christoph Schell, Manuel Rogg and Tobias B. Huber. "Combining these findings with primary podocyte culture systems helped us to identify a central role for actomyosin machinery in podocyte foot process development and showed that ARP3 is also involved in controlling the adhesion and mechanoadaptation of podocytes."

On the basis of the hypothesis that the adhesion machinery of podocytes is in tight interplay with cytoskeletal components, Schell and colleagues used a proteomics approach to identify involvement of N-WASPARP2-ARP3 - a complex that is involved in the nucleation and assembly of branched actin networks - in both biological processes. To assess the relevance of N-WASP-ARP2ARP3-generated actin networks to podocyte development, the researchers used a series of conditional knockout mouse models and primary podocyte culture systems to show that loss of either N-WASP or ARP3 disturbed the development of podocyte foot processes. Cells deficient in Arp3 showed distorted focal adhesions characterized by a hypermaturated, plaque-like appearance, associated with overactivation of actomyosin machinery and increased inherent tension. These cells were also unable to adapt to mechanical stress, promoting podocyte detachment. "We can now more precisely describe the events leading to foot process effacement," says Huber.

"We aim to assess how this understanding can be used to predict and halt podocyte cytoskeletal alterations in glomerular diseases."

Susan J. Allison

ORIGINAL ARTICLE Schell, C. et al. ARP3 controls the podocyte architecture at the kidney filtration barrier. Dev. Cell https://doi.org/10.1016/j.devcel.2018.11.011 (2018)

\title{
TRANSPLANTATION
}

\section{Transcriptional programmes of kidney injury}

Understanding the molecular mechanisms associated with the progression of acute to chronic kidney injury is important for the identification of patients at risk of chronic kidney disease (CKD). Building on previous work showing that the transition from acute kidney injury to irreversible renal damage in mice follows a defined transcriptional programme, researchers now show that similar biological processes occur in humans. "We identified two main transcriptional programmes associated with progressive transition to chronic injury or with apparent recovery of the kidney," says Pietro Cippà. "The transition to chronic injury was unexpectedly similar in human and mouse, suggesting that the fundamental transcriptional programme associated with CKD might be uniform across species."

To study changes in transcriptional programmes in response to renal injury in humans, Cippà and colleagues performed transcriptome analysis of 163 protocol biopsy samples from 42 kidney transplant recipients at four time points using computational tools originally developed for single-cell RNA sequencing. "We took advantage of machine learning techniques specifically developed to characterize single cell heterogeneity in complex tissues to study 'single patient' heterogeneity in a complex clinical setting, and generated a global map of kidney injury progression from the first minutes after reperfusion to fibrosis," explains Cippà. Their analyses showed a rapid upregulation of immediate-early genes involved in stress responses following reperfusion in all patients, after which transcriptional signatures diverged in accordance with progression to kidney injury or renal recovery. "Our study represents a starting point for many potential applications," says Cippà. "Although the predictive relevance of our data needs to be verified and more focused analyses of the underlying biology are required, use of such strategies might help identify fundamental cellular processes that underlie CKD and define novel therapeutic strategies."

Susan J. Allison

ORIGINAL ARTICLE Cippà, P. E. et al. Transcriptional trajectorie of human kidney injury progression.JCl Insight https://doi.org/ 10.1172/jci.insight.123151 (2018)

\section{ACUTE KIDNEY INJURY}

\section{Sepsis halts renal protein translation}

Sepsis can cause multiple organ failure and is associated with high patient mortality, but how is sepsis linked to widespread organ damage? Takashi Hato, Pierre Dagher and colleagues now report that bacterial sepsis triggers a cellular antiviral response in the kidney; the effects of this response on protein translation potentially lead to organ failure.

The researchers injected lipopolysaccharide (LPS) into mice to induce moderate endotoxaemia, which resulted in severe acute kidney injury. Proximal tubule cells respond to LPS via Toll-like receptor 4 (TLR4) and the researchers used ribosome profiling to establish the ribosome footprint of septic kidneys and assess changes in the kidney translatome over time.

LPS injection induced rapid and transient nuclear factor- $\kappa \mathrm{B}$ (NF- $\kappa \mathrm{B}$ ) activation, which led to a rise in pro-inflammatory cytokines, such as IL-1 and IL-6, in the early stages of sepsis (that is, 4 hours after LPS challenge). Moreover, interferon and interferon-induced antiviral genes were upregulated at this stage and their translation was sustained for up to 28 hours. In contrast to this early increase in protein levels, in late sepsis (that is, 16 hours after LPS challenge) the researchers observed a global decline in protein synthesis. "Protein translation shutdown was preceded by the upregulation of double-stranded RNA-activated protein kinase (Eif2ak2)," adds Hato. In response to viral infections, Eif2ak2 upregulation blocks protein translation in an attempt to prevent viral replication. These effects on translation might directly lead to organ failure, as restoring protein translation was renoprotective in this model.

EIF2AK2 expression was also increased in kidney biopsy samples from patients with acute tubular necrosis related to bacterial sepsis. "Our examination of tissues from patients with sepsis supports a model in humans that is similar to what is observed in mice, although the timescale is potentially different," explains Dagher.

"Our work links inflammation in the early stages of sepsis to late-stage organ failure at the molecular level," concludes Dagher.

Monica Wang

ORIGINAL ARTICLE Hato, T. et al. Bacterial sepsis triggers an antiviral response that causes translation shutdown. J. Clin. Invest. https://doi.org/10.1172/JCl123284 (2018) 